\title{
ON FINITE DIVISION RINGS
}

\author{
ROBERT H. OEHMKE
}

\begin{abstract}
Herein it is shown that the set of right powers of a generic element of a finite division ring contains a basis of the ring as an algebra over a prime field. This result is then applied to finite flexible division rings of characteristic not 2 to obtain commutativity.
\end{abstract}

A finite division ring (or semifield [4]) is a finite algebraic system containing at least two distinguished elements 0 and 1 . A division ring $\mathfrak{A}$ possesses two binary operations, addition and multiplication, designated in the usual notation and satisfying the following axioms:

(i) $(\mathfrak{A},+)$ is a group with identity 0 .

(ii) If $a, b \in \mathfrak{A}$ and $a b=0$ then $a=0$ or $b=0$.

(iii) If $a, b, c \in \mathfrak{U}$ then $a(b+c)=a b+a c$ and $(a+b) c=a c+b c$.

(iv) The element 1 satisfies the relationship $1 \cdot a=a \cdot 1=a$ for all $a$ in $\mathfrak{X}$.

It is seen easily that there are unique solutions to the equations $a x=b$ and $x a=b$ for every nonzero $a$ and every $b$ in $\mathfrak{A}$. It also follows easily that addition is commutative. In fact it can be seen that $\mathfrak{A}$ is a vector space over some prime field $F=G F(p)$ and that $\mathfrak{A}$ has $p^{n}$ elements where $n$ is the dimension of $\mathfrak{A}$ over $F$ [4].

Let $u_{1}, \ldots, u_{n}$ be a basis of $\mathfrak{A}$ over $F$ and let $\delta_{1}, \ldots, \delta_{n}$ be a set of $n$ algebraically independent elements over $F$. Let $K=F\left(\delta_{1}, \ldots, \delta_{n}\right)$. As usual the algebra $\mathfrak{A}_{K}$ is the tensor product over $F$ of $\mathfrak{A}$ and $K . \mathfrak{A}_{K}$ can be considered as a vector space over $K$ with basis $u_{1}, \ldots, u_{n}$. Thus

$$
x=\delta_{1} u_{1}+\cdots+\delta_{n} u_{n}
$$

is an element of $\mathfrak{A}_{K}$ and is called the generic element of $\mathfrak{A}$ [3].

For any $y$ in $\mathfrak{A}_{K}$ we shall write $y^{l}=y^{l-1} \cdot y, y^{0}=1$ and $y^{1}=y$ if $l>2$ for the right powers of the element $y$. If $R_{y}$ designates the linear transformation induced by right multiplication by $y$ then $y^{l}=1 \cdot R_{y}^{l}$. If $x_{0}$ is an element of $\mathfrak{A}$ and is written as $x_{0}=\delta_{10} u_{1}+\cdots+\delta_{n 0} u_{n}$ where $\delta_{i 0} \in F$ we shall call $x_{0}$ a specialization of $x$.

Now $R_{x}$ as a linear transformation on the vector space $\mathfrak{A}_{K}$ over $K$ has a characteristic polynomial $m_{x}(\lambda)$ of degree $n$. If $S_{x}$ is the matrix representation of $R_{x}$ with respect to the basis $u_{1}, \ldots, u_{n}$ then $m_{x}(\lambda)=\left|\lambda I-S_{x}\right|$. But clearly, $S_{x}$ has entries that are linear over $F$ in the variables $\delta_{1}, \ldots, \delta_{n}$. Thus $m_{x}(\lambda)$ is a homogeneous polynomial in $F\left[\lambda, \delta_{1}, \ldots, \delta_{n}\right]$. We can write $m_{x}(\lambda)=\sum \sigma_{i}(x) \lambda^{i}$ where $\sigma_{i}(x)$ is a homogeneous polynomial in $F\left[\delta_{1}, \ldots, \delta_{n}\right]$ of degree $n-i$.

We let $R_{x_{0}}$ designate the linear transformation induced by right multiplication by $x_{0}$ in $\mathfrak{A}$. Then $R_{x_{0}}$ is represented by the matrix $S_{x_{0}}$ with respect to the basis

Received by the editors May 11, 1979.

AMS (MOS) subject classifications (1970). Primary 17A01, 51A05.

(c) 1980 American Mathematical Society 0002-9939/80/0000-0253/\$01.75 
$u_{1}, \ldots, u_{n}$ where $S_{x_{0}}$ is the specialization of $S_{x}$; i.e., where the variables $\delta_{1}, \ldots, \delta_{n}$ occurring in $S_{x}$ are replaced by $\delta_{10}, \ldots, \delta_{n 0}$. Since the entries of $S_{x}$ are polynomials this specialization is always well defined. We further extend $K$ to a field $L$ which contains a new variable $\delta$ and the roots of the polynomials $m_{x_{0}}(\lambda)$ and $\delta-\mu_{x_{0}}(\lambda)$ in $\lambda$ where $\mu_{x_{0}}(\lambda)$ is the minimal polynomial satisfied by $R_{x_{0}}$. We can write

$$
m_{x_{0}}(\lambda)=\prod_{j}\left(\lambda-\xi_{j}\right), \quad \delta-\mu_{x_{0}}(\lambda)=\prod_{i}\left(\lambda-\beta_{i}\right) .
$$

We borrow generously from Braun and Koecher [2, p. 102] in proving the following result.

THEOREM 1. The irreducible factors of $\mu_{x_{0}}(\lambda)$ are the same as the irreducible factors of $m_{x_{0}}(\lambda)$.

Proof.

$$
\begin{aligned}
\prod_{j}\left(\delta-\mu_{x_{0}}\left(\xi_{j}\right)\right) & =\prod_{j} \prod_{i}\left(\xi_{j}-\beta_{i}\right)= \pm \prod_{i} \prod_{j}\left(\beta_{i}-\xi_{j}\right) \\
& = \pm \prod_{i}\left|\beta_{i} I-S_{x_{0}}\right|= \pm\left|\prod_{i}\left(\beta_{i} I-S_{x_{0}}\right)\right| \\
& = \pm\left|\prod_{i}\left(S_{x_{0}}-\beta_{i} I\right)\right|=\left|\delta I-\mu\left(S_{x_{0}}\right)\right|
\end{aligned}
$$

But $\mu\left(S_{x_{0}}\right)=0$. Therefore the above polynomial in $\delta$ is $\delta^{n}$. Therefore $\mu_{x_{0}}\left(\xi_{j}\right)=0$ for all $\xi_{j}$ and the theorem follows.

Now $R_{x_{0}}$ is nonsingular on the vector space $\mathfrak{A}$ due to the definition of a division ring. Thus none of the $\xi_{j}$ 's can be 0 . It now follows that the constant term of $m_{x}(\lambda)$ is a homogeneous form in $\delta_{1}, \ldots, \delta_{n}$ not representing 0 nontrivially.

Let $V_{x}$ be the subspace of $\mathfrak{A}_{K}$ generated by the right powers of $x$ over $K$ and $\bar{R}_{x}$ the restriction of $R_{x}$ to $V_{x}$. Assume $V_{x}$ is of dimension $m$. The characteristic polynomial, $\varepsilon_{x}(\lambda)$, of $\bar{R}_{x}$ is of degree $m$ and must divide $m_{x}(\lambda)$. Therefore $\varepsilon_{x}(\lambda)$ must be homogeneous in $\lambda, \delta_{1}, \ldots, \delta_{n}$ and hence is a polynomial in $\lambda$ with polynomial coefficients in the $\delta_{i}$ 's [5, p. 127]. Therefore the "constant term" of $\varepsilon_{x}(\lambda)$ is a form of degree $m$ in $\delta_{1}, \ldots, \delta_{m}$ that divides the "constant term" of $m_{x}(\lambda)$. But then the "constant term" of $\varepsilon_{x}(\lambda)$ is a form in $n$ variables and of degree $m$ that does not represent 0 nontrivially. By the Artin-Chevalley Theorem [5, p. 140] we see that $m=n$. Therefore

THEOREM 2. $V_{x}=\mathfrak{A}_{K}$ and $1, x, \ldots, x^{n-1}$ is a basis of $\mathfrak{A}_{K}$.

Following a similar argument as above and assuming $\mathfrak{A}$ is strictly power associative, McCrimmon [6] gave an alternate proof to Albert's result [1] that such I's were fields. We shall give another application of this result to flexible algebras.

A flexible algebra is an algebra for which $a(b a)=(a b) a$ for all $a$ and $b$ in $\mathfrak{A}$. If $L_{a}$ and $R_{a}$ denote the linear transformations induced by left multiplication and right multiplication respectively then the flexible identity is equivalent to $R_{a} L_{a}=$ $L_{a} R_{a}$ for all $a \in \mathfrak{A}$. The flexible identity can be linearized to

$$
a(b c)+c(b a)=(a b) c+(c b) a \text {. }
$$


If the characteristic is not 2 then (1) implies $a(b a)=(a b) a$. Thus in the characteristic not 2 case the flexible identity extends to all scalar extensions of $\mathfrak{A}$, in particular to $\mathfrak{A}_{K}$. In $\mathfrak{A}_{K}$ let $W$ be the set of all vectors $w$ such that $w\left(R_{x}-L_{x}\right)=0$. Clearly $1 \in W$. If $\mathfrak{A}$ is flexible then $W$ is an $R_{x}$ invariant subspace of $\mathfrak{A}$ and must be $V_{x}$. But $V_{x}=\mathfrak{A}_{K}$. Therefore

TheOrem 3. If $\mathfrak{A}$ is a flexible division ring of characteristic not 2 then $\mathfrak{A}$ is commutative.

\section{REFERENCES}

1. A. A. Albert, On nonassociative division algebras, Trans. Amer. Math. Soc. 72 (1952), 296-309.

2. H. Braun and M. Koecher, Jordan-Algebren, Springer-Verlag, Berlin, 1966.

3. N. Jacobson, Structure and representations of Jordan algebras, Amer. Math. Soc. Colloq. Publ., vol. 39, Amer. Math. Soc., Providence, R. I., 1968.

4. D. Knuth, Finite semifields and projective planes, J. Algebra 2 (1965), 182-217.

5. S. Lang, Algebra, Addison-Wesley, Reading, Mass., 1965.

6. K. McCrimmon, A note on finite division rings, Proc. Amer. Math. Soc. 17 (1966), 1173-1177.

Department of Mathematics, The University of Iowa, Iowa Crty, Iowa 52242 